\title{
RISCO PARA SÍNDROME DE BURNOUT EM PROFESSORES DE ESCOLAS PÚBLICAS DE MACAÉ - RJ*
}

Luana Silva Monteiro ${ }^{1}$

ORCID: https://orcid.org/0000-0003-3599-6947

Naiara Sperandio ${ }^{2}$

ORCID: http://orcid.org/0000-0002-9015-3849

Jaína Chumacker Frez $^{3}$

ORCID: http://orcid.org/0000-0002-9615-5036

Larissa Costa Ferreira Viveiros ${ }^{4}$

ORCID: http://orcid.org/0000-0003-1691-4109

Walquiria Terezinha Oliveira Rodrigues ${ }^{5}$

ORCID: http://orcid.org/0000-0003-4831-3658

Ana Eliza Port Lourenço ${ }^{6}$

ORCID: https://orcid.org/0000-0002-9619-8052

Priscila Vieira Pontes ${ }^{7}$

ORCID: https://orcid.org/0000-0003-3914-9307

Recebido em: 31/07/2021.

Publicado em: 0/09/2021.

\section{RESUMO}

Este estudo objetivou avaliar o risco para o desenvolvimento da Síndrome de Burnout (SB) em professores da rede pública de ensino de Macaé, Rio de Janeiro. Trata-se de um estudo transversal realizado em 2019 com professores de quatro escolas públicas de Macaé. Os participantes responderam um questionário autopreenchível sobre características sociodemográficas, laborais e de saúde. Para avaliar o risco da SB, utilizou-se como referência o "Cuestionario para la Evaluación del Síndrome de Quemarse por el Trabajo", validado para profissionais da educação no Brasil. Foi calculado um escore para as respostas dos professores em cada uma das quatro dimensões desse questionário. Escores baixos $(<2)$

\footnotetext{
${ }^{1}$ Núcleo de Estudos em Saúde e Nutrição na Escola - NESANE, Campus UFRJ Macaé.

${ }^{2}$ Núcleo de Estudos em Saúde e Nutrição na Escola - NESANE, Campus UFRJ Macaé.

${ }^{3}$ Núcleo de Estudos em Saúde e Nutrição na Escola - NESANE, Campus UFRJ Macaé.

${ }^{4}$ Núcleo de Estudos em Saúde e Nutrição na Escola - NESANE, Campus UFRJ Macaé.

${ }^{5}$ Núcleo de Estudos em Saúde e Nutrição na Escola - NESANE, Campus UFRJ Macaé.

${ }^{6}$ Professora de Nutrição e Saúde Coletiva na Universidade Federal do Rio de Janeiro, Campus Macaé.

${ }^{7}$ Núcleo de Estudos em Saúde e Nutrição na Escola - NESANE, Campus UFRJ Macaé.
} 
na dimensão de Ilusão pelo trabalho, e escores altos $(\geq 2)$ nas dimensões de Desgaste psíquico, Indolência e Culpa permitiram inferir o risco para SB. Foram avaliados 84 professores (25 do ensino fundamental e 59 do ensino médio), com média de idade de 44 anos, a maioria do sexo feminino $(76,2 \%)$ e com excesso de peso $(69,9 \%)$. Somente no ensino médio foram observados professores com risco para SB (3,0\%). Contudo, parcela elevada dos avaliados apresentou escores críticos para Desgaste psíquico $(69,0 \%)$ e Culpa (32,1\%). Características dos professores, como "ser mulher", "não ser casado", "ter menor tempo de atuação no magistério" e "ter hipertensão" implicaram em maior presença de escores críticos para SB. Apesar de uma pequena parcela dos professores ter sido identificada com risco para SB, muitos apresentaram escore elevado para Desgaste psíquico e Culpa, o que denota que a SB pode, cedo ou tarde, vir a acometer um maior número de professores. Para além da contribuição científica, esses resultados podem ser usados para promover reflexão e direcionar políticas e ações locais acerca da saúde dos professores em Macaé.

Palavras-chaves: síndrome de Burnout; professores; saúde.

\title{
RISK FOR BURNOUT SYNDROME IN TEACHERS OF PUBLIC SCHOOLS IN MACAÉ - RJ
}

\begin{abstract}
This study aimed to assess the risk for the development of Burnout Syndrome (BS) among teachers of public schools of Macaé - a Brazilian municipality in the northern region of the state of Rio de Janeiro. This is a cross-sectional study carried out in 2019 with teachers from four public schools of Macaé. The participants answered a self-administered questionnaire about their sociodemographic characteristics, labor, and health. The risk of developing BS was evaluated by the "Cuestionario para la Evaluación del Syndrome de Quemarse por el Trabajo", which was validated for education professionals in Brazil. We calculated a score for the teachersee answers in each of the four dimensions of this questionnaire. Low scores $(<2)$ in the dimension Illusion for work, and high scores $(\geq 2)$ in the dimensions Psychic exhaustion, Indolence and Guilt allowed us to infer the risk for BS. We evaluated 84 teachers (25 from elementary and 59 from high school), mean age of 44 years. Most of them were women $(76.2 \%)$ and had excess weight (69.9\%). Only high school teachers were identified at risk for BS (3.0\%). However, a large proportion of the participants had critical scores for Psychic exhaustion $(69.0 \%)$ and Guilt (32.1\%). "Being a woman", "not being married", "working as a teacher for a shorter time" and "having hypertension" implied a greater presence of critical scores for BS. Although only a small number of teachers were at risk for BS, many of them had high scores for Psychic exhaustion and Guilt. This means that the SB can, soon or later, reach a greater number of teachers. Beyond scientific contributions, these findings may be useful to promote reflection and guide local policies and actions related to Macaé teachers ${ }^{\text {ee }}$ health.
\end{abstract}

Key words: Burnout syndrome; teachers. health. 


\section{RIESGO DE SÍNDROME DE BURNOUT EN PROFESORES DE ESCUELAS PÚBLICAS DE MACAÉ - RJ}

\section{RESUMEN}

Este estudio tuvo como objetivo evaluar el riesgo de desarrollo del Síndrome de Burnout (SB) en profesores de escuelas públicas de la ciudad de Macaé, Río de Janeiro. Se trata de un estudio transversal realizado en 2019 con profesores de cuatro escuelas públicas de Macaé. Los participantes respondieron un cuestionario autoadministrado sobre características sociodemográficas, ocupacionales y de salud. Para evaluar el riesgo de SB se utilizó el "Cuestionario para la Evaluación del Síndrome de Quemarse por el Trabajo" validado para profesionales de la educación en Brasil. Se calculó un puntaje para las respuestas de los profesores en cada una de las cuatro dimensiones de este cuestionario. Puntajes bajos $(<2)$ en la dimensión Ilusión para el trabajo, y puntajes altos $(\geq 2)$ en las dimensiones Agotamiento psíquico, Indolencia y Culpabilidad nos permitieron inferir el riesgo de SB. Se evaluó a 84 profesores ( 25 de primaria y 59 de escuela secundaria), con edad media de 44 años. La mayoría de ellos eran mujeres $(76,2 \%)$ y tenían sobrepeso $(69,9 \%)$. Solo en la escuela secundaria se observaron profesores en riesgo de SB (3,0\%). Sin embargo, una gran parte de los evaluados tenía puntajes críticos de Desgaste psíquico $(69,0 \%)$ y Culpabilidad $(32,1 \%)$. Características de los profesores, como "ser mujer", "no estar casada", "trabajar como profesor por un tiempo más corto" y "tener hipertensión" implicaron una mayor presencia de puntujes críticos para BS. Aunque solo una pequeña parte de los profesores estaban en riesgo de SB, muchos obtuvieron puntajes altos en Desgaste psíquico y Culpa, lo que denota que SB puede, tarde o temprano, afectar a un mayor número de profesores. Además de la contribución científica, estos resultados se pueden utilizar para promover la reflexión y orientar las políticas y acciones locales sobre la salud de los profesores en Macaé.

Palabras clave: Síndrome de Burnout. maestros. salud.

\section{INTRODUÇÃO}

O trabalho demanda grande parte do tempo de cada indivíduo, às vezes os privando do convívio social. Nem sempre o trabalho está associado a sentimentos bons e a realização profissional, podendo causar insatisfação, fadiga e esgotamento. Estudos científicos revelam que quando a saúde do trabalhador está insatisfatória, o absenteísmo é favorecido, impactando tanto o profissional, quanto o empregador (BATISTA et al., 2010; FRANÇA et al., 2014).

Dentre as ocupações laborais relacionadas ao desgaste profissional, destacam-se aquelas de caráter assistencial, como profissionais de saúde, segurança pública e professores. Nessas profissões há elevada pressão, exigência emocional e conflitos, bem como pouco reconhecimento (ARVIDSSON et al., 2016; WILHELM; ZANELLI, 2014). Um dos principais fatores de desgaste é a ausência do senso de coletividade nas organizações, que 
pode ser expresso por deficiência na qualidade das interações interpessoais, presença constante de conflitos e dificuldades no trabalho em equipe (FRANÇA et al., 2014).

Assim, o magistério pode ser considerado altamente estressante, seja na educação básica ou no ensino universitário. Devido ao acúmulo de atribuições, ocorre sobrecarga de trabalho e estresse, com possíveis repercussões sobre a saúde e desempenho profissional (BATISTA et al., 2011, CARVALHO, 2002; CUNHA, 2009). A categoria docente do ensino fundamental e médio é considerada uma das mais expostas a enfermidades relacionadas ao estresse, como a Síndrome de Burnout (SB) (CARLOTTO; PALAZZO, 2006).

Burnout é uma expressão em inglês que refere "àquilo que deixou de funcionar por absoluta falta de energia" (FREUDENBERGER, 1974). A SB resulta do estresse crônico advindo do cotidiano laboral, como no magistério (FRANÇA et al., 2014). Os fatores estressantes no exercício do magistério incluem ritmo intenso, longa jornada de trabalho, exigências burocráticas, dificuldades de relação interpessoal, dentre outros (ASSUNÇÃO; ABREU, 2019; PADILLA; THOMPSON, 2016; GASPARINI; BARRETO; ASSUNÇÃO, 2005). Os professores podem ficar desmotivados, devido à falta de tempo para atividades de qualificação profissional, e para atividades fora do trabalho que gerem prazer (CARLOTTO; PALAZZO, 2006; RAUSCH; DUBIELLA, 2013). Tal situação pode impactar negativamente a qualidade de vida e a saúde dos professores, além de reduzir a própria capacidade laboral (PEREIRA et al., 2014; OLIVEIRA FILHO A; NETTO-OLIVEIRA; OLIVEIRA, 2012).

É fundamental, portanto, investigar as condições de saúde dos professores, para fins de promover estratégias de intervenção e prevenção do estresse e do desgaste profissional. Tal relevância é ampliada ao se considerar o papel-chave desempenhado pelos professores e pela educação no desenvolvimento das sociedades. Nesse sentido, o presente estudo tem como objetivo avaliar o risco para o desenvolvimento da SB em professores da rede pública de ensino do município de Macaé, Rio de Janeiro.

\section{MÉTODOS}

Foi realizado um estudo transversal com professores de quatro escolas da rede pública de ensino de Macaé, Rio de Janeiro, sendo duas de ensino fundamental e duas de ensino médio, selecionadas por conveniência. Não foram elegíveis professores licenciados, afastados, gestantes ou lactantes. 
A coleta de dados ocorreu em 2019, por equipe treinada. Foi aplicado um questionário autopreenchível, sobre características sociodemográficas (sexo, idade, situação marital e números de filhos), laborais (tempo no exercício no magistério), de saúde (condição de peso, diabetes, hipertensão arterial e hipercolesterolemia) e de risco para a SB.

As medidas de peso e estatura foram autorreferidas e utilizadas para o cálculo do Índice de Massa Corporal (IMC). Indivíduos com IMC igual ou superior a $25 \mathrm{~kg} / \mathrm{m}^{2}$ foram classificados com excesso de peso (OMS, 1998). Foram obtidas informações sobre o diagnóstico prévio de hipertensão arterial, diabetes e hipercolesterolemia por meio das seguintes perguntas: “Atualmente, o(a) Sr.(a) está tomando algum medicamento para controlar a pressão alta?", “Algum MÉDICO já lhe disse que o(a) Sr.(a) tem diabetes?, “Algum MÉDICO já lhe disse que o(a) Sr.(a) tem colesterol elevado?"

Para a avaliação sociodemográfica, a variável idade foi categorizada em professores com menos de 45 anos e com 45 anos ou mais. O tempo de magistério dos professores foi agrupado em menos de 15 anos e com 15 anos ou mais de atuação. A variável número de filhos foi transformada em uma variável dicotômica (tem filhos ou não tem).

$\mathrm{O}$ risco para o desenvolvimento da SB foi avaliado tendo como referência o "Cuestionario para la Evaluacióndel Síndrome de Quemarse por el Trabajo" (CESQT-PE), validado para profissionais da educação no Brasil (GIL-MONTE; CARLOTTO; CÂMARA, 2010). Esse questionário inclui 20 perguntas que avaliam situações e sentimentos, com opções de resposta que variam de 0 (nunca) a 4 (muito frequente: todos os dias). O questionário é composto por quatro dimensões, a saber: Ilusão pelo trabalho (cinco perguntas), Desgaste psíquico (quatro perguntas), Indolência (seis perguntas) e Culpa (cinco perguntas). Para cada dimensão foi calculado um escore a partir da média da pontuação alcançada nas respostas. Escores baixos $(<2)$ na dimensão Ilusão pelo trabalho, e escores altos $(\geq 2)$ nas dimensões de Desgaste psíquico, Indolência e Culpa permitiram inferir o risco para a SB (GIL-MONTE; CARLOTTO; CÂMARA, 2010; SIMÕES, 2014).

Os dados foram analisados no pacote estatístico Statistical Package for the Social Sciences-SPSS, versão 19. As variáveis contínuas foram descritas por média, desvio padrão (DP); e as variáveis categóricas por proporção (\%). As variáveis contínuas foram testadas por meio do teste de Kolgomorov-Smirnov para verificar se os dados possuíam distribuição simétrica. Para testar as diferenças entre variáveis contínuas, foi usado o teste t de student; para variáveis categóricas, o teste Exato de Fisher. Considerou-se 5\% de significância estatística (pvalor $<0,05)$. 
O projeto foi aprovado pelo Comitê de Ética em Pesquisa do Hospital Clementino Fraga Filho (CAAE: 49258313.1.0000.5257). Todos os professores participantes assinaram o Termo de Consentimento Livre e Esclarecido e foram informados sobre os objetivos, procedimentos, benefícios e potenciais riscos envolvidos com a sua participação no estudo.

\section{RESULTADOS}

Foram avaliados 84 docentes, sendo 25 do ensino fundamental e 59 do ensino médio. A média de idade foi de 44 anos ( $\mathrm{DP}=10,1)$ (fundamental: 47 anos vs. médio: 43 anos; p- valor= 0,088). A maior parcela dos professores $(76,2 \%)$ era formada por mulheres (fundamental: 92,0\% vs. médio: 69,5\%; p-valor=0,028); indivíduos com filhos (71,4\%) (fundamental: 88,0\% vs. médio: 64,4\%; p-valor=0,035); e casados (63,1\%) (fundamental: 64,0\% vs. médio: 62,7\%; p-valor=1,00). O tempo médio de atuação no magistério foi de 17 anos $(\mathrm{DP}=8,7)$ (fundamental: 14,9 anos vs. médio: 17,6 anos; p-valor=0,207), sendo que 53,6\% dos professores possuíam menos de 15 anos de magistério (fundamental: 60,0\% vs. médio: 50,8\%; p-valor=0,324) (Tabela $1)$.

Segundo o diagnóstico médico prévio referido pelos professores, 26,2\% eram hipertensos, 10,7\% tinham hipercolesterolemia e 7,1\% diabetes. Em relação à condição de peso, 69,0\% dos professores apresentaram excesso de peso. Não foram observadas diferenças estatísticas nas variáveis de saúde segundo o segmento escolar (Tabela 2).

A média de escore foi igual a 3,0 para a dimensão Ilusão, 2,2 Desgaste Psíquico, 1,2 Indolência e 1,5 Culpa. Essas pontuações foram mais elevadas nos professores do ensino fundamental quando comparados aos do ensino médio: Ilusão (4,0 vs. 2,6; p-valor=0,00), Desgaste Psíquico (2,7 vs. 2,0; p-valor=0,00), Indolência (1,6 vs. 1,0; p-valor=0,00) e Culpa $(2,0$ vs. 1,$3 ; \mathrm{p}$-valor=0,00). A média do escore na dimensão Indolência foi maior entre os professores não casados, do que entre os casados (1,4 vs. 1,1; p-valor=0,023). Professores com menor tempo de magistério, quando comparados com os de mais tempo, apresentaram maior escore médio na dimensão Culpa (1,7 vs. 1,3; p-valor=0,046) e Indolência, apesar de que nessa, a significância estatística foi borderline (1,3 vs. 1,0; p-valor=0,051) (Tabela 3).

Os professores com excesso de peso apresentaram escore médio na dimensão Culpa (1,6 vs.1,2; -valor=0,037) mais elevado quando comparados com aqueles sem excesso de peso, independentemente do segmento escolar. Destaca-se que no ensino fundamental, o 
escore médio na dimensão Culpa também foi mais elevado entre os professores com hipertensão arterial quando comparados aos sem hipertensão (2,4 vs.1,8; p-valor=0,02) (Tabela 3).

A maioria dos professores apresentava-se com ilusão pelo trabalho $(86,9 \%)$, sendo essa proporção mais elevada entre aqueles do ensino fundamental quando comparados aos do ensino médio (100\% vs. 81,4\%; p-valor=0,02). Entretanto, a maior parte dos professores $(69,0 \%)$ apresentava-se com desgaste psíquico, principalmente no ensino fundamental (fundamental: 88\% vs. médio: 61\%; p-valor=0,01). Além disso, respectivamente 10,7\% e $32,1 \%$ dos professores apresentaram escore elevado nas dimensões Indolência (fundamental: $24 \%$ vs. médio: $5,1 \%$; p-valor=0,01) e Culpa ( $52 \%$ vs. $23,4 \%$; p-valor=0,02), com maior proporção no ensino fundamental (Tabela 4).

Destaca-se que a frequência de desgaste foi maior entre professores do sexo feminino do que masculino $(78,1 \%$ vs. $40 \%$; p-valor=0,001). A proporção de escores elevados para Indolência foi maior entre professores não casados do que entre casados $(25,8 \%$ vs. 1,9\%; pvalor $=0,001)$, independente do segmento de ensino. Entre os professores com hipertensão observou-se maior percentual de escores elevados para a dimensão Culpa (50\% vs. 25,8\%; pvalor $=0,037)$ (Tabela 4).

Um percentual de 34,5\% dos professores apresentou escore alterado em ao menos uma das dimensões estudadas; e 40,5\% em duas ou mais dimensões. De uma forma geral, a frequência de dimensões alteradas foi superior entre mulheres do que entre homens $(p=0,010)$ (Tabela 5). Adicionalmente, os professores apresentaram em média, 1,3 (DP=0,9) dimensões alteradas para o risco de SB, com valores mais elevados no ensino fundamental (fundamental: 1,6 vs. médio: 1,$1 ; \mathrm{p}$-valor $=0,013)$.

Avaliando o conjunto das dimensões, não foram observados professores com risco para a SB no ensino fundamental. Já no ensino médio, dois professores $(3,0 \%)$ apresentaram- se em risco. 
Tabela 1 - Características sociodemográficas e laborais de professores de quatro escolas da rede pública de Macaé do ensino fundamental e médio ( $\mathrm{n}=84)$. Macaé, 2019.

\begin{tabular}{|c|c|c|c|c|c|c|c|}
\hline \multirow[b]{2}{*}{ Características } & \multicolumn{2}{|c|}{ Total } & \multicolumn{2}{|c|}{$\begin{array}{c}\text { Ensino } \\
\text { Fundamental }\end{array}$} & \multicolumn{2}{|c|}{ Ensino Médio } & \multirow[t]{2}{*}{ p-valor* } \\
\hline & $\mathrm{n}$ & $\%$ & & & & & \\
\hline Total & 84 & 100 & 25 & 29,8 & 59 & 70,2 & \\
\hline \multicolumn{8}{|l|}{ Sexo } \\
\hline Masculino & 20 & 23,8 & 2 & 8,0 & 18 & 30,5 & 0,028 \\
\hline Feminino & 64 & 76,2 & 23 & 92,0 & 41 & 69,5 & \\
\hline \multicolumn{8}{|l|}{ Faixa etária } \\
\hline$<45$ anos & 44 & 52,4 & 11 & 44,0 & 33 & 55,9 & 0,471 \\
\hline$\geq 45$ anos & 39 & 46,4 & 13 & 52,0 & 26 & 44,1 & \\
\hline Sem informação & 1 & 1,2 & 1 & 4,0 & - & - & \\
\hline \multicolumn{8}{|l|}{ Situação marital } \\
\hline Casado & 53 & 63,1 & 16 & 64,0 & 37 & 62,7 & 1,00 \\
\hline Outro & 31 & 36,9 & 9 & 36,0 & 22 & 37,3 & \\
\hline \multicolumn{8}{|l|}{ Filhos } \\
\hline Sim & 60 & 71,4 & 22 & 88,0 & 38 & 64,4 & $\mathbf{0 , 0 3 5}$ \\
\hline Não & 24 & 28,6 & 3 & 12,0 & 21 & 35,6 & \\
\hline \multicolumn{8}{|c|}{ Tempo de trabalho no magistério } \\
\hline$<15$ anos & 45 & 53,6 & 15 & 60,0 & 30 & 50,8 & 0,324 \\
\hline$\geq 15$ anos & 37 & 44,0 & 8 & 32,0 & 29 & 49,2 & \\
\hline Sem informação & 2 & 2,4 & 2 & 8,0 & - & - & \\
\hline
\end{tabular}

*Teste Exato de Fisher - p-valor <0,05

Fonte: Elaboração Própria com dados obtidos na investigação.

Tabela 2 - Características de saúde de professores de quatro escolas da rede pública de Macaé do ensino fundamental e médio ( $\mathrm{n=84}$ ). Macaé, RJ, 2019.

\begin{tabular}{lccccccc}
\hline & \multicolumn{2}{c}{ Total } & \multicolumn{2}{c}{$\begin{array}{c}\text { Ensino } \\
\text { Fundamental }\end{array}$} & Ensino Médio & p-valor* \\
\hline Características & $\mathrm{n}$ & $\%$ & & & & & \\
\hline Total & 84 & 100 & 25 & 29,8 & 59 & 70,2 & \\
Hipertensão Arterial & 22 & 26,2 & 9 & 36,0 & 13 & 22,0 & 0,277 \\
Sim & 62 & 73,8 & 16 & 64,0 & 46 & 78,0 & \\
Não & 6 & 7,1 & 0 & 0 & 6 & 10,2 & 0,119 \\
Diabetes & 78 & 92,9 & 25 & 100 & 53 & 89,8 & \\
Sim & & & & & & & \\
Não & 9 & 10,7 & 4 & 16,0 & 5 & 8,5 & 0,440 \\
Hipercolesterolemia & 75 & 89,3 & 21 & 84,0 & 54 & 91,5 & \\
Sim & & & & & & & \\
Não & 22 & 26,2 & 9 & 36,0 & 13 & 22,0 & 0,089 \\
Condição de peso & 58 & 69,0 & 12 & 48,0 & 46 & 78,0 & \\
$\quad$ Sem excesso de peso & 4 & 4,8 & 4 & 16,0 & - & - & \\
$\quad$ Com excesso de peso & Sem informação & & & & & & \\
\hline
\end{tabular}

Fonte: Elaboração própria com dados obtidos na investigação. * Teste Exato de Fisher - p-valor $<0,05$ 
Tabela 3 - Médias dos escores das dimensões de risco para Síndrome de Burnout ${ }^{1}$ em professores de quatro escolas da rede pública de Macaé do ensino fundamental e médio, segundo características sociodemográficas, laborais e de saúde (n=84). Macaé, RJ, 2019.

\begin{tabular}{|c|c|c|c|c|c|c|c|c|c|c|c|c|}
\hline \multirow[b]{3}{*}{ Características } & \multicolumn{12}{|c|}{ Escores (média) das dimensões da Síndrome de Burnout $^{1}$} \\
\hline & \multicolumn{4}{|c|}{ Total $(n=84)$} & \multicolumn{4}{|c|}{ Ensino Fundamental $(\mathrm{n}=25)$} & \multicolumn{4}{|c|}{ Ensino Médio $(\mathrm{n}=59)$} \\
\hline & Ilusão & Desgaste & Indolência & Culpa & Ilusão & Desgaste & Indolência & Culpa & Ilusão & Desgaste & Indolência & Culpa \\
\hline & \multicolumn{4}{|c|}{ Média (Desvio padrão) } & \multicolumn{4}{|c|}{ Média (Desvio padrão) } & \multicolumn{4}{|c|}{ Média (Desvio padrão) } \\
\hline Total & $3,0(1,0)$ & $2,2(0,8)$ & $1,2(0,7)$ & $1,5(0,8)$ & $4,0\left(0,7^{) a}\right.$ & $2,7(0,6)^{a}$ & $1,6(0,5)^{a}$ & $2,0(0,7)^{\mathrm{a}}$ & $2,6(0,8)$ & $2,0(0,8)$ & $1,0(0,6)$ & $1,3(0,7)$ \\
\hline $\begin{array}{l}\text { Sexo } \\
\text { Masculino }\end{array}$ & & & & & & & & & & & & \\
\hline Feminino & $\begin{array}{l}2,9(1,0) \\
3,1(0,9)\end{array}$ & $\begin{array}{l}1,9(1,0) \\
2,3(07)\end{array}$ & $1,0(0,7)$ & $1,4(0,7)$ & $\begin{array}{l}4,2(0,8)^{a} \\
40(07)^{a}\end{array}$ & $2,6(1,2)$ & $\begin{array}{l}1,7(0,7) \\
1,5(0,5) \text { a }\end{array}$ & $2,4(0)^{a}$ & $2,7(0,9)$ & $1,8(1,0)$ & $0,9(0,6)$ & $1,3(0,7)$ \\
\hline p-valor* & 0,393 & $\begin{array}{c}2,3(0,7) \\
0,059\end{array}$ & $\begin{array}{c}1,2(0,7) \\
0,249\end{array}$ & $\begin{array}{c}1,0(0,8) \\
0,535\end{array}$ & $\begin{array}{c}4,0(0, /) \\
0,74\end{array}$ & $\begin{array}{c}2, /(0,0) \\
0,84\end{array}$ & $\begin{array}{c}1,5(0,5)^{a} \\
0,76\end{array}$ & $\begin{array}{c}1,9(0, /) \\
0,42\end{array}$ & $\begin{array}{c}2,6(0,7) \\
0,475\end{array}$ & $\begin{array}{c}2,1(0,7) \\
0,270\end{array}$ & $\begin{array}{c}1,0(0,7) \\
0,608\end{array}$ & $\begin{array}{c}1,3(0,7) \\
0,994\end{array}$ \\
\hline \multicolumn{13}{|l|}{ Faixa etária } \\
\hline$<45$ anos & $3,1(1,0)$ & $2,2(0,9)$ & $1,2(0,6)$ & $1,5(0,8)$ & $4,2(0,7)^{\mathrm{a}}$ & $2,7(0,7)^{\mathrm{a}}$ & $1,5(0,4)$ & $2,1(0,8)^{\mathrm{a}}$ & $2,7(0,7)$ & $2,1(0,9)$ & $1,1(0,6)$ & $1,2(0,7)$ \\
\hline$\geq 45$ anos & $3,0(0,9)$ & $2,2(0,8)$ & $1,1(0,7)$ & $1,6(0,7)$ & $3,9(0,6)^{\mathrm{a}}$ & $2,7(0,6)^{\mathrm{a}}$ & $1,6(0,6)^{\mathrm{a}}$ & $1,9(0,6)$ & $2,5(0,8)$ & $1,9(0,8)$ & $0,9(0,7)$ & $1,4(0,7)$ \\
\hline p-valor* & 0,755 & 0,98 & 0,502 & 0,490 & 0,23 & 0,88 & 0,47 & 0,40 & 0,540 & 0,752 & 0,113 & 0,272 \\
\hline \multicolumn{13}{|l|}{ Situação marital } \\
\hline Casado & $3,1(1,0)$ & $2,2(0,8)$ & $1,1(0,5)$ & $1,6(0,8)$ & $4,1(0,8)^{\mathrm{a}}$ & $2,6(0,7)^{\mathrm{a}}$ & $1,4(0,4)^{\mathrm{a}}$ & $2,0(0,7)^{\mathrm{a}}$ & $2,6(0,7)$ & $2,0(0,7)$ & $0,9(0,5)$ & $1,4(0,8)$ \\
\hline Outro & $3,0(1,0)$ & $2,3(0,9)$ & $1,4(0,8)$ & $1,5(0,7)$ & $3,9(0,5)^{\mathrm{a}}$ & $2,9(0,5)^{\mathrm{a}}$ & $1,8(0,6)$ & $1,9(0,6)^{\mathrm{a}}$ & $2,6(0,9)$ & $2,0(0,9)$ & $1,2(0,8)$ & $1,3(0,6)$ \\
\hline p-valor* & 0,690 & 0,77 & $\mathbf{0 , 0 2 3}$ & 0,571 & 0,38 & 0,28 & 0,06 & 0,53 & 0,994 & 0,898 & 0,071 & 0,788 \\
\hline \multicolumn{13}{|l|}{ Ter filho } \\
\hline Sim & $3,1(1,0)$ & $2,3(0,8)$ & $1,2(0,6)$ & $1,6(0,8)$ & $4,1(0,7)^{\mathrm{a}}$ & $2,7(0,6)^{\mathrm{a}}$ & $1,6(0,5)^{\mathrm{a}}$ & $2,0(0,7)^{\mathrm{a}}$ & $2,6(0,8)$ & $2,0(0,8)$ & $0,9(0,6)$ & $1,3(0,6)$ \\
\hline Não & $2,8(0,9)$ & $2,1(0,9)$ & $1,2(0,7)$ & $1,5(0,8)$ & $3,8(0,5)^{\mathrm{a}}$ & $2,6(0,4)$ & $1,6(0,8)$ & $1,7(0,6)$ & $2,6(0,9)$ & $2,0(0,9)$ & $1,1(0,7)$ & $1,5(0,8)$ \\
\hline p-valor* & 0,128 & 0,417 & 0,905 & 0,707 & 0,50 & 0,71 & 0,99 & 0,38 & 0,895 & 0,904 & 0,314 & 0,313 \\
\hline \multicolumn{13}{|l|}{ Tempo de trabalho } \\
\hline$<15$ anos & $3,1(1,0)$ & $2,3(0,9)$ & $1,3(0,6)$ & $1,7(0,8)$ & $4,2(0,7)^{\mathrm{a}}$ & $2,7(0,7)^{\mathrm{a}}$ & $1,5(0,5)$ & $2,2(0,8)^{\mathrm{a}}$ & $2,6(0,8)$ & $2,1(0,9)$ & $1,2(0,6)$ & $1,4(0,6)$ \\
\hline$\geq 15$ anos & $2,9(0,9)$ & $2,2(0,8)$ & $1,0(0,7)$ & $1,3(0,8)$ & $3,9(0,6)^{\mathrm{a}}$ & $2,7(0,6)^{\mathrm{a}}$ & $1,6(0,5)^{\mathrm{a}}$ & $1,9(0,6)$ & $2,6(0,7)$ & $2,0(0,8)$ & $0,9(0,7)$ & $1,2(0,8)$ \\
\hline p-valor* & 0,584 & 0,479 & 0,051 & 0,046 & 0,22 & 0,96 & 0,45 & 0,33 & 0,921 & 0,516 & 0,132 & 0,205 \\
\hline \multicolumn{13}{|l|}{ Condição de peso } \\
\hline Sem excesso de peso & $3,1(1,2)$ & $2,3(0,8)$ & $1,2(0,8)$ & $1,2(0,7)$ & $3,9(0,7)^{\mathrm{a}}$ & $2,7(0,8)^{\mathrm{a}}$ & $1,6(0,6)^{\mathrm{a}}$ & $1,6(0,5)^{\mathrm{a}}$ & $2,5(1,1)$ & $1,9(0,7)$ & $0,9(0,9)$ & $0,9(0,7)$ \\
\hline Com excesso de peso & $2,9(0,9)$ & $2,2(0,9)$ & $1,2(0,6)$ & $1,6(0,8)$ & $3,9(0,7)$ & $2,7(0,5)^{\mathrm{a}}$ & $1,6(0,6)^{\mathrm{a}}$ & $2,3(0,8)^{\mathrm{a}}$ & $2,7(0,7)$ & $2,1(0,9)$ & $1,1(0,6)$ & $1,4(0,7)$ \\
\hline p-valor* & 0,543 & 0,924 & 0,795 & $\mathbf{0 , 0 3 7}$ & 0,97 & 0,84 & 0,83 & $\mathbf{0 , 0 5 0}$ & 0,447 & 0,543 & 0,299 & $\mathbf{0 , 0 1 6}$ \\
\hline \multicolumn{13}{|l|}{ Hipertensão Arterial } \\
\hline Sim & $3,3(0,9)$ & $2,3(0,8)$ & $1,2(0,7)$ & $1,8(0,9)$ & $3,9(0,9)^{\mathrm{a}}$ & $2,8\left(0,5^{\mathrm{a}}\right)$ & $1,7(0,6)^{\mathrm{a}}$ & $2,4(0,8)^{\mathrm{a}}$ & $2,9(0,6)$ & $1,9(0,7)$ & $0,9(0,7)$ & $1,4(0,7)$ \\
\hline Não & $2,9(1,0)$ & $2,2(0,9)$ & $1,2(0,6)$ & $1,4(0,7)$ & $4,1(0,6)^{\mathrm{a}}$ & $2,7(0,7)^{\mathrm{a}}$ & $1,4(0,5)^{\mathrm{a}}$ & $1,8(0,6)^{\mathrm{a}}$ & $2,5(0,8)$ & $2,1(0,9)$ & $1,1(0,7)$ & $1,3(0,7)$ \\
\hline p-valor* & 0,127 & 0,908 & 0,859 & 0,065 & 0,61 & 0,57 & 0,19 & $\mathbf{0 , 0 2 0}$ & 0,169 & 0,427 & 0,238 & 0,901 \\
\hline \multicolumn{13}{|l|}{ Diabetes } \\
\hline Sim & $2,8(0,5)$ & $1,8(0,5)$ & $0,9(0,5)$ & $1,5(0,7)$ & - & - & - & - & $2,8(0,5)$ & $1,8(0,5)$ & $0,990,5)$ & $1,5(0,7)$ \\
\hline Não & $3,1(1,0)$ & $2,3(0,8)$ & $1,2(0,7)$ & $1,5(0,8)$ & $4,0(0,7)^{\mathrm{a}}$ & $2,7(0,6)^{\mathrm{a}}$ & $1,6(0,5)^{\mathrm{a}}$ & $2,0(0,7)^{\mathrm{a}}$ & $2,6(0,8)$ & $2,1(0,9)$ & $1,1(0,7)$ & $1,3(0,7)$ \\
\hline p-valor* & 0,549 & 0,181 & 0,447 & 1,00 & - & - & - & - & 0,539 & 0,467 & 0,854 & 0,463 \\
\hline \multicolumn{13}{|l|}{ Hipercolesterolemia } \\
\hline Sim & $3,5(0,9)$ & $2,4(0,6)$ & $1,1(0,4)$ & $1,4(0,8)$ & $4,2(0,7)^{\mathrm{a}}$ & $2,5(0,8)$ & $1,3(0,2)$ & $1,7(0,5)$ & $2,9(0,8)$ & $2,3(0,5)$ & $0,9(0,4)$ & $1,1(0,9)$ \\
\hline Não & $2,9(0,9)$ & $2,2(0,9)$ & $1,2(0,7)$ & $1,6(0,8)$ & $4,0(0,7)^{\mathrm{a}}$ & $2,8(0,6)^{\mathrm{a}}$ & $1,6(0,5)^{\mathrm{a}}$ & $2,0(0,7)^{\mathrm{a}}$ & $2,6(0,8)$ & $2,0(0,9)$ & $1,1(0,7)$ & $1,4(0,7)$ \\
\hline p-valor* & 0,176 & 0,554 & 0,681 & 0,522 & 0,83 & 0,43 & 0,45 & 0,40 & 0,363 & 0,454 & 0,688 & 0,484 \\
\hline
\end{tabular}


Tabela 4 - Frequência de escores de risco ${ }^{1}$ nas dimensões Ilusão pelo trabalho, Desgaste psíquico, Indolência e Culpa para o risco de Síndrome de Burnout ${ }^{2}$ em professores de quatro escolas da rede pública de Macaé do ensino fundamental e médio, segundo características sociodemográficas, laborais e de saúde (n=84). Macaé, RJ, 2019.

\begin{tabular}{|c|c|c|c|c|c|c|c|c|c|c|c|c|}
\hline \multirow[b]{3}{*}{ Características } & \multicolumn{12}{|c|}{ Frequência (\%) das pontuações consideradas de risco ${ }^{1}$ para Síndrome de Burnout - n (\%) } \\
\hline & \multicolumn{4}{|c|}{ Total $(\mathrm{n}=84)$} & \multicolumn{4}{|c|}{ Ensino Fundamental $(\mathrm{n}=25)$} & \multicolumn{4}{|c|}{ Ensino Médio $(\mathrm{n}=59)$} \\
\hline & Ilusão & Desgaste & Indolência & Culpa & Ilusão & Desgaste & Indolência & Culpa & Ilusão & Desgaste & Indolência & Culpa \\
\hline Total & $73(86,9)$ & $58(69,0)$ & $9(10,7)$ & $27(32,1)$ & $25(100)^{\mathrm{a}}$ & $22(88)^{a}$ & $6(24,0)^{\mathrm{a}}$ & $13(52)^{a}$ & $48(81,4)$ & $36(61,0)$ & $3(5,1)$ & $14(23,7$ \\
\hline \multicolumn{13}{|l|}{ Sexo } \\
\hline Masculino & $17(85,0)$ & $8(40,0)$ & $2(10,0)$ & $6(30,0)$ & $2(100)$ & $1(50)$ & $1(50)^{\mathrm{a}}$ & $2(100)^{a}$ & $15(83,3)$ & $7(38,9)$ & $1(5,6)$ & $4(22,2)$ \\
\hline Feminino & $56(87,6)$ & $50(78,1)$ & $7(10,9)$ & $21(32,8)$ & $23(100)^{a}$ & $22(91,3)^{a}$ & $5(21,7)^{\mathrm{a}}$ & $11(47,8)^{a}$ & $33(80,5)$ & $29(70,7)$ & $2(4,9)$ & $10(24,4$ \\
\hline p-valor* & 0,772 & 0,001 & 0,906 & 0,814 & - & 0,085 & 0,369 & 0,157 & 0,796 & $\mathbf{0 , 0 2 1}$ & 0,913 & 0,857 \\
\hline \multicolumn{13}{|l|}{ Faixa etária } \\
\hline$<45$ anos & $39(88,6)$ & $29(65,9)$ & $3(6,8)$ & $13(29,5)$ & $11(100)$ & $9(81,8)$ & $1(9,1)$ & $6(54,5)^{a}$ & $28(84,8)$ & $20(60,6)$ & $2(6,1)$ & $7(21,2)$ \\
\hline$\geq 45$ anos & $33(84,6)$ & $28(71,8)$ & $6(15,4)$ & $14(35,9)$ & $13(100)$ & $12(92,3)^{\mathrm{a}}$ & $5(38,5)^{a}$ & $7(53,8)$ & $20(76,9)$ & $16(61,5)$ & $1(3,8)$ & $7(26,9)$ \\
\hline p-valor* & 0,590 & 0,564 & 0,210 & 0,538 & - & 0,439 & 0,098 & 0,973 & 0,438 & 0,942 & 0,701 & 0,609 \\
\hline \multicolumn{13}{|l|}{ Situação marital } \\
\hline Casado & $45(84,9)$ & $36(67,9)$ & $1(1,9)$ & $19(35,8)$ & $16(100)^{\mathrm{a}}$ & $13(81,3)$ & $1(6,3)$ & $9(56,3)^{a}$ & $29(78,4)$ & $23(62,2)$ & 0 & $10(27,0$ \\
\hline Outro & $28(90,3)$ & $22(71,0)$ & $8(25,8)$ & $8(35,8)$ & $9(100)$ & $9(100)^{a}$ & $5(55,6)^{\mathrm{a}}$ & $4(44,4)$ & $19(86,4)$ & $13(59,1)$ & $3(13,6)$ & $4(18,2)$ \\
\hline p-valor* & 0,478 & 0,771 & 0,001 & 0,342 & -- & 0,166 & 0,006 & 0,571 & 0,446 & 0,815 & 0,021 & 0,440 \\
\hline \multicolumn{13}{|l|}{ Ter Filho } \\
\hline Sim & $52(86,7)$ & $42(70,0)$ & $6(10,0)$ & $18(30,0)$ & $22(100)^{a}$ & $19(86,4)^{\mathrm{a}}$ & $5(22,7)^{a}$ & $12(54,5)^{\mathrm{a}}$ & $30(78,9)$ & $23(60,5)$ & $1(2,6)$ & $6(15,8)$ \\
\hline Não & $21(87,5)$ & $16(66,7)$ & $3(12,5)$ & $9(37,5)$ & 0 & 0 & $1(33,3)$ & $1(33,3)$ & $18(85,7)$ & $13(61,9)$ & $2(9,5)$ & $8(38,1)$ \\
\hline p-valor* & 0,919 & 0,765 & 0,738 & 0,506 & - & 0,495 & 0,687 & 0,490 & 0,523 & 0,917 & 0,249 & 0,054 \\
\hline \multicolumn{13}{|l|}{ Tempo de trabalho } \\
\hline$<15$ anos & $39(86,7)$ & $32(71,1)$ & $7(15,6)$ & $18(40,0)$ & $15(100)$ & $12(80,0)$ & $5(33,3)^{a}$ & $9(60,0)^{a}$ & $24(80,0)$ & $20(66,7)$ & $2(6,7)$ & $9(30,0)$ \\
\hline$\geq 15$ anos & $32(86,5)$ & $24(64,9)$ & $2(5,4)$ & $8(21,6)$ & $8(100)$ & $8(100)^{a}$ & $1(12,5)$ & $3(37,5)$ & $24(82,8)$ & $16(55,2)$ & $1(3,4)$ & $5(17,2)$ \\
\hline p-valor* & 0,981 & 0,545 & 0,143 & 0,075 & - & 0,175 & 0,278 & 0,304 & 0,786 & 0,365 & 0,574 & 0,249 \\
\hline \multicolumn{13}{|l|}{ Condição de peso } \\
\hline Sem excesso de peso & $19(86,4)$ & $16(72,7)$ & $4(18,2)$ & $4(18,2)$ & $9(100)$ & $7(77,8)$ & $3(33,3)$ & $3(33,3)$ & $10(76,9)$ & $9(69,2)$ & $1(7,7)$ & $1(7,7)$ \\
\hline Com excesso de peso & $50(86,2)$ & $38(65,5)$ & $5(8,6)$ & $5(8,6)$ & $12(100)$ & $11(91,7)^{\mathrm{a}}$ & $3(25,0)^{\mathrm{a}}$ & $8(66,7)^{\mathrm{a}}$ & $38(82,6)$ & $27(58,7)$ & $2(4,3)$ & $13(28,3$ \\
\hline p-valor* & 0,985 & 0,539 & 0,227 & 0,120 & - & 0,368 & 0,676 & 0,130 & 0,642 & 0,492 & 0,628 & 0,124 \\
\hline \multicolumn{13}{|l|}{ Hipertensão Arterial } \\
\hline Sim & $21(95,5)$ & $17(77,3)$ & $3(13,6)$ & $11(50,0)$ & $9(100)$ & $13(81,3)^{\mathrm{a}}$ & $3(18,8)^{a}$ & $6(37,5)^{a}$ & $12(92,3)$ & $8(61,5)$ & 0 & $4(30,8)$ \\
\hline Não & $52(83,9)$ & $41(66,1)$ & $6(9,7)$ & $16(25,8)$ & $16(100)^{a}$ & $9(100)$ & $3(33,3)$ & $7(77,8)$ & $36(78,3)$ & $28(60,9)$ & $3(6,5)$ & $10(21,7$ \\
\hline p-valor* & 0,166 & 0,331 & 0,606 & $\mathbf{0 , 0 3 7}$ & - & 0,166 & 0,412 & 0,053 & 0,251 & 0,965 & 0,345 & 0,499 \\
\hline \multicolumn{13}{|l|}{ Diabetes } \\
\hline Sim & $6(100)$ & $3(50,0)$ & 0 & $2(33,3)$ & 0 & 0 & 0 & 0 & $6(100)$ & $3(50,0)$ & 0 & $2(33,3)$ \\
\hline Não & $67(85,9)$ & $55(70,5)$ & $9(11,5)$ & $25(32,1)$ & $25(100)^{a}$ & $22(88,0)^{\mathrm{a}}$ & $6(24,0)^{\mathrm{a}}$ & $13(52,0)^{\mathrm{a}}$ & $42(79,2)$ & $33(62,3)$ & $3(5,7)$ & $12(22,6$ \\
\hline p-valor* & 0,324 & 0,295 & 0,379 & 0,948 & - & - & - & - & 0,216 & 0,559 & 0,505 & 0,560 \\
\hline \multicolumn{13}{|l|}{ Hipercolesterolemia } \\
\hline Sim & $8(88,9)$ & $6(66,7)$ & 0 & $3(33,3)$ & $4(100)$ & $3(75,0)$ & 0 & $11(52,4)$ & $3(60,0)$ & $4(80,0)$ & 0 & $1(20,0)$ \\
\hline Não & $65(86,7)$ & $52(69,3)$ & $9(12,0)$ & $24(32,0)$ & $21(100)^{a}$ & $19(90,5)^{\mathrm{a}}$ & $6(28,6)^{\mathrm{a}}$ & $2(50,0)^{a}$ & $33(61,1)$ & $44(81,5)$ & $3(5,6)$ & $13(24,1$ \\
\hline p-valor* & 0,852 & 0,870 & 0,271 & 0,935 & - & 0,383 & 0,220 & 0,930 & 0,935 & 0,961 & 0,589 & 0,838 \\
\hline
\end{tabular}

Fonte: Elaboração própria. Dimensão Ilusão pelo trabalho (escore $<2)$ e Dimensões Desgaste psíquico, Indolência e Culpa (escore $\geq 2) ;{ }^{2}$ Avaliada pelo Cuestionario para la Evaluación del Síndrome de Quemarse por el Trabajo; *Teste Exato de Fisher (p-valor <0,05); ${ }^{a}$ Comparação das médias dos escores das dimensões de risco para a Síndrome de Burnout por segmento escolar. 
Tabela 5 - Número de dimensões alteradas para o risco de Síndrome de Burnout ${ }^{1}$ em professores de quatro escolas da rede pública de Macaé do ensino fundamental e médio, segundo características sociodemográficas, laborais e de saúde (n=84). Macaé, RJ, 2019.

\begin{tabular}{|c|c|c|c|c|c|c|c|c|c|}
\hline \multirow[b]{3}{*}{ Características } & \multicolumn{9}{|c|}{ Número de dimensões da Síndrome de Burnout alteradas - n (\%) } \\
\hline & \multicolumn{3}{|c|}{ Total $(\mathrm{n}=84)$} & \multicolumn{3}{|c|}{ Ensino Fundamental $(\mathrm{n}=25)$} & \multicolumn{3}{|c|}{ Ensino Médio (n=59) } \\
\hline & Zero & 1 & $\geq 2$ & Zero & 1 & $\geq 2$ & Zero & 1 & $\geq 2$ \\
\hline Total & $21(25,0)$ & $29(34,5)$ & $34(40,5)$ & $2(8,0)$ & $10(40,0)$ & $13(52,0)$ & $19(32,2)$ & $19(32,2)$ & $21(35,6)$ \\
\hline \multicolumn{10}{|l|}{ Sexo } \\
\hline Masculino & $10(50,0)$ & $3(15,0)$ & $7(35,0)$ & 0 & $1(50,0)$ & $1(50,0)$ & $10(55,6)$ & $2(11,1)$ & $6(33,3)$ \\
\hline Feminino & $11(17,2)$ & $26(40,6)$ & $27(42,2)$ & $2(8,7)$ & $9(39,1)$ & $12(52,2)$ & $9(22,0)$ & $17(41,5)$ & $15(36,6)$ \\
\hline p-valor* & & 0,010 & & & 1,0 & & & 0,019 & \\
\hline \multicolumn{10}{|l|}{ Faixa etária } \\
\hline$<45$ anos & $12(27,3)$ & $17(38,6)$ & $15(34,1)$ & $1(9,1)$ & $5(45,5)$ & $5(45,5)$ & $11(33,3)$ & $12(36,4)$ & $10(30,3)$ \\
\hline$\geq 45$ anos & $9(23,1)$ & $11(28,2)$ & $19(48,7)$ & $1(7,7)$ & $4(30,8)$ & $8(61,5)$ & $8(30,8)$ & $7(26,9)$ & $11(42,3)$ \\
\hline p-valor* & & 0,428 & & & 0,827 & & & 0,578 & \\
\hline \multicolumn{10}{|l|}{ Situação marital } \\
\hline Casado & $12(22,6)$ & $20(37,7)$ & $21(39,6)$ & $2(12,5)$ & $6(37,5)$ & $8(50,0)$ & $10(27)$ & $14(37,8)$ & $13(35,1)$ \\
\hline Outro & $9(29,0)$ & $9(29,0)$ & $13(41,9)$ & 0 & $4(44,4)$ & $5(55,6)$ & $9(40,9)$ & $5(22,7)$ & $8(36,4)$ \\
\hline p-valor* & & 0,683 & & & 0,702 & & & 0,418 & \\
\hline \multicolumn{10}{|l|}{ Ter filho } \\
\hline $\operatorname{Sim}$ & $13(21,7)$ & $25(41,7)$ & $22(36,7)$ & $2(9,1)$ & $8(36,4)$ & $12(54,5)$ & $11(28,9)$ & $17(44,7)$ & $10(26,3)$ \\
\hline Não & $8(33,3)$ & $4(16,7)$ & $12(50,0)$ & 0 & $2(66,7)$ & $1(33,3)$ & $8(38,1)$ & $2(9,5)$ & $11(52,4)$ \\
\hline p-valor* & & 0,089 & & & 0,661 & & & 0,014 & \\
\hline \multicolumn{10}{|l|}{ Tempo de trabalho } \\
\hline$<15$ anos & $8(17,8)$ & $18(40,0)$ & $19(42,2)$ & $2(13,3)$ & $5(33,3)$ & $8(53,3)$ & $6(20,0)$ & $13(43,3)$ & $11(36,7)$ \\
\hline$\geq 15$ anos & $13(35,1)$ & $10(27,0)$ & $14(37,8)$ & 0 & $4(50,0)$ & $4(50,0)$ & $13(44,8)$ & $6(20,7)$ & $10(34,5)$ \\
\hline p-valor* & & 0,180 & & & 0,695 & & & 0,077 & \\
\hline \multicolumn{10}{|l|}{ Condição de peso } \\
\hline Sem excesso de peso & $6(27,3)$ & $8(36,4)$ & $8(38,4)$ & $2(22,2)$ & $3(33,3)$ & $4(44,4)$ & $4(30,8)$ & $5(38,5)$ & $4(30,8)$ \\
\hline Com excesso de peso & $15(25,9)$ & $19(32,8)$ & $24(41,4)$ & 0 & $5(41,7)$ & $7(58,3)$ & $15(32,6)$ & $14(30,4)$ & $17(37,0)$ \\
\hline p-valor* & & 0,950 & & & 0,381 & & & 0,924 & \\
\hline \multicolumn{10}{|l|}{ Hipertensão Arterial } \\
\hline Sim & $4(18,2)$ & $7(31,8)$ & $11(50,0)$ & 0 & $2(22,2)$ & $7(77,8)$ & $4(30,8)$ & $5(38,5)$ & $4(30,8)$ \\
\hline Não & $17(27,4)$ & $22(35,5)$ & $23(37,1)$ & $2(12,5)$ & $8(50,0)$ & $6(37,5)$ & $15(32,6)$ & $14(30,4)$ & $17(37,0)$ \\
\hline p-valor* & & 0,514 & & & 0,192 & & & 0,924 & \\
\hline \multicolumn{10}{|l|}{ Diabetes } \\
\hline Sim & $2(33,3)$ & $3(50,0)$ & $1(16,7)$ & 0 & 0 & 0 & $2(33,3)$ & $3(50,0)$ & $1(16,7)$ \\
\hline Não & $19(24,4)$ & $26,(33,3)$ & $33(42,3)$ & $2(8,0)$ & $10(40,0)$ & $13(52,0)$ & $17(32,1)$ & $16(30,2)$ & $20(37,7)$ \\
\hline p-valor* & & 0,492 & & & - & & 0,500 & & \\
\hline \multicolumn{10}{|l|}{ Hipercolesterolemia } \\
\hline Sim & $3(33,3)$ & $2(22,2)$ & $4(44,4)$ & $1(25,0)$ & $1(25,0)$ & $2(50,0)$ & $2(40,0)$ & $1(20,0)$ & $2(40,0)$ \\
\hline Não & $18(24,0)$ & $27(36,0)$ & $30(40,0)$ & $1(4,8)$ & $9(42,9)$ & $11(52,4)$ & $17(31,5)$ & $18(33,3)$ & $19(35,2)$ \\
\hline p-valor* & & 0,743 & & & 0,496 & & & 1,00 & \\
\hline
\end{tabular}




\section{DISCUSSÃO}

Dentre as quatro dimensões avaliadas acerca do risco para a SB, a Ilusão pelo trabalho foi a que apresentou resultados mais positivos. Aproximadamente, 87\% dos professores avaliados não estavam com escores críticos nessa dimensão. Em contrapartida, a dimensão Desgaste psíquico foi a mais comprometida. Foram observados valores críticos nessa dimensão em mais de 3/5 dos professores, com maior frequência de no ensino fundamental. Chama atenção também nesse segmento de ensino que metade dos professores apresentou valores críticos na dimensão Culpa. Adicionalmente, observou-se que algumas características dos professores implicaram em maior presença de escores críticos. Isso aconteceu com "ser mulher" para a dimensão de Desgaste psíquico, "não ser casado" para Indolência, "ter menor tempo de atuação no magistério" para Indolência e Culpa, e "ter hipertensão arterial" para Culpa.

Ainda que uma parcela dos professores tenha ilusão pelo trabalho e o risco para a SB tenha sido observado apenas em pequena parcela dos professores do ensino médio, vale ressaltar que a SB é um processo lento, gradativo e progressivo, que pode evoluir em anos ou décadas, sendo difícil a sua identificação nos estágios iniciais (CARLOTTO, 2011). Nesse sentido, o fato de um percentual elevado da população estudada apresentar escores críticos para Desgaste psíquico e Culpa pode já significar um impacto negativo no desempenho profissional e na saúde. Isso pois, comprometimentos na dimensão Desgaste psíquico sugerem que o indivíduo está se tornando esgotado fisicamente e mentalmente devido às atividades laborais; e na dimensão Culpa, sinaliza um sentimento atrelado ao comportamento inadequado praticado junto àqueles com quem trabalha (GIL-MONTE; CARLOTTO; CÂMARA, 2010). Assim, caso haja persistência destes estressores, cedo ou tarde, a SB pode surgir entre os professores.

A conjuntura da profissão docente no Brasil abarca múltiplas exigências profissionais, baixos salários e desvalorização. Soma-se ainda o fato de algumas escolas estarem inseridas em contextos violentos e de faltarem recursos pedagógicos. Tal cenário pode gerar insatisfação em quem leciona, diminuir seu encanto e contentamento pelo trabalho, favorecendo o cansaço ou até mesmo o abandono da profissão (ARAÚJO et al. 2019). O elevado desgaste psíquico observado neste estudo pode ter relação com as características da profissão. Os fatores estressantes da profissão podem estar contribuindo de forma negativa 
com a saúde dos professores, sinalizando uma relação íntima entre o trabalho e o desgaste (SISTO; SANTOS; NORONHA, 2008). Friedrich et al. (2015) evidenciaram o estresse ocupacional como uma reação que se caracteriza por tensão, frustração, ansiedade e exaustão emocional, devido a aspectos da modernidade.

Uma pesquisa realizada pela Confederação Nacional de Trabalhadores da Educação e pela Universidade de Brasília avaliou as condições de trabalho e de saúde mental, destacando a SB. Essa pesquisa incluiu 52 mil trabalhadores da educação de 1.440 escolas públicas do Brasil. Os resultados foram preocupantes, uma vez que $26 \%$ dos professores apresentaram exaustão emocional, associada à desvalorização profissional, baixa autoestima e ausência de resultados percebidos no trabalho (ARAÚJO, 2019). Carlotto e Palazzo (2006) avaliaram 217 professores de nível fundamental e médio de escolas particulares da região metropolitana de Porto Alegre e apontaram que a carga de trabalho exacerbada, o elevado número e comportamento de alunos foram algumas variáveis relacionadas ao desgaste profissional.

Carlotto e colaboradores (2012) desenvolveram um estudo com 63 professores de escolas especiais da região central do Estado do Rio Grande do Sul com o objetivo de identificar a prevalência da SB e a sua associação com fatores de risco psicossociais. Os autores identificaram 30,6\% de professores com um nível moderado e 14,3\% com uma forma mais severa da SB. O nível moderado foi associado ao aumento da iniquidade; já o mais severo foi associado à sobrecarga de trabalho, iniquidade, insatisfação laboral e problemas de saúde. Citase também a pesquisa realizada por Goulart e Lipp (2008) que identificou o estresse entre 175 professoras do ensino fundamental de escolas públicas do interior do Estado de São Paulo através do Inventário de estresse para adultos. Nesse estudo, 56,6\% das professoras estavam estressadas, apresentando desgaste, cansaço constante, tensão muscular, problemas de memória, irritabilidade, angústia e ansiedade.

Cabe destacar que a população do presente estudo foi majoritariamente do sexo feminino. Atualmente, as mulheres têm exercido dupla ou até mesmo tripla jornada de trabalho. Uma parcela expressiva das mulheres é responsável pelo cuidado dos familiares, execução de tarefas domésticas, além do seu trabalho profissional, o que pode impactar sua qualidade de vida. Acrescenta-se que as mulheres ainda enfrentam barreiras para ter valorização no mercado de trabalho se comparadas aos homens (KAN; SULLIVAN; GERSHUNY, 2011; GUIRALDELLI, 2012; CONNELL, 2013). Zibetti e Pereira (2010) observaram que mulheres que atuavam no magistério tinham uma maior sobrecarga devido a questões adversas, como a falta de tempo para descanso e a necessidade contínua de terminar atividades da escola em casa. 
As atividades de cuidar e ensinar frequentemente têm sido atreladas as mulheres e revelam que as relações de gênero são importantes para entender também as associações entre pressão laboral, vida em casal e ter ou não filhos (ASSUNÇÃO, 2019). O magistério por vezes ainda é visto como uma ampliação da função materna. Nessa concepção, a exaustão emocional teria relação com o universo feminino e diferenças de construção social de gênero deveriam ser consideradas no processo de adoecimento da categoria docente (CARLOTTO, 2011; CARLOTTO et al., 2014).

Em relação ao menor tempo de magistério ter impactado de forma negativa no presente estudo para as dimensões Indolência e Culpa, alguns estudos já sinalizaram essa relação (CARLOTTO, 2011; GAVISH; FRIEDMAN, 2010). Friedrich e colaboradores (2015) destacaram que a vulnerabilidade dos mais jovens ao estresse ocorre devido à transição para o mundo adulto e ao enfrentamento de situações novas, como a busca por uma profissão e por estabilidade. Já professores que atuam há mais tempo na profissão tornam-se referência, encontram menor dificuldade para lidar com situações de sala de aula, se sentem menos pressionados e constroem um vínculo forte com a instituição de ensino (KOETZ; REMPEL; PÉRICO, 2013).

O presente estudo observou que grande parte dos professores possuía agravos como excesso de peso, hipertensão e hipercolesterolemia. De acordo com Assunção e colaboradores (2019), as inúmeras tarefas destinadas aos docentes, concomitantemente com as demandas extras ao trabalho, podem contribuir para a diminuição de tempo para o autocuidado e de lazer com os amigos e a família. Tais desequilíbrios comprometem o repouso e podem levar a prejuízos à saúde. Franco e Monteiro (2016) comentam que o estresse ocupacional pode levar ao aumento do consumo de álcool e drogas, baixa produtividade, insatisfação, diminuição do comprometimento, contribuindo para o afastamento do trabalho e desfavorecendo a saúde.

Outro ponto observado no presente estudo foi que professores com excesso de peso se sentiam mais culpados quando comparados aos sem excesso de peso. Essa maior culpa pode ter origens além da sala de aula. A mídia dispõe de diversos discursos, muitas vezes infundados sobre o sobrepeso, trazendo sentimentos negativos a quem se encontra nessa condição (NOGUEIRA, 2001). Oliveira e colaboradores (2012) identificaram em jornal de grande circulação discursos como "pessoas gordas não se cuidam" e "ser saudável e se cuidar é ser magro", sugerindo uma culpabilização das pessoas que apresentam excesso de peso. Os sentimentos de culpa podem ser ratificados por discursos de saúde equivocados ou pela dificuldade de se adequar a um corpo magro (MATTOS; LUZ, 2009). 
Uma limitação do presente estudo relaciona-se ao tamanho amostral, cujos dados não podem ser extrapolados para outras populações. Contudo, estudos que realizam o diagnóstico situacional a nível local são de extrema importância para traçar o perfil epidemiológico e contribuir para a vigilância em saúde e para o desenvolvimento de ações focadas nas demandas do território. Assim, os olhares das produções científicas mais próximas da comunidade podem fortalecer e orientar políticas e ações locais. A gestão municipal de Macaé pode, portanto, utilizar os estudos científicos realizados no município para desenvolver ações alinhadas com as necessidades locais.

Nessa linha, a escola ganha destaque como um espaço privilegiado para ações de promoção em saúde com foco nos professores, por ser esse o local que esses profissionais passam grande parte do seu dia. Os professores são protagonistas no cenário escolar e desempenham papéis que muitas vezes vão além do ensino. Conssitem em profissionais que devem ser valorizados e necessitam de uma maior atenção quanto a sua saúde física e mental, pois essa interferirá diretamente na sua qualidade de vida e na qualidade de ensino e formação dos seus alunos.

Diante dos resultados obtidos é importante fomentar estratégias para auxiliar os professores do município de Macaé a diminuir as fontes geradoras de estresse e tornar o ambiente de trabalho um espaço promotor de saúde. Assim, destaca-se a busca do equilíbrio entre o indivíduo e o trabalho, sendo necessário enfocar tanto o funcionário quanto o ambiente em que este desenvolve suas atividades, além de buscar resoluções no contexto social do local de trabalho.

Intervenções devem ser elaboradas considerando os diferentes níveis em que o fenômeno estudado se manifesta, assim como as variáveis que o afetam. Nem sempre intervenções focais podem ser feitas dado a presença de estressores contextuais, que se apresentam pouco manejáveis pelos professores, como salários baixos. Sugere-se realizar intervenções em saúde com planejamento de longo prazo, por meio de abordagem compreensiva, sem julgamentos e imposições, de maneira a olhar o trabalho e os sujeitos de forma integral e humanizada.

\section{CONSIDERAÇÕES FINAIS}

A avaliação dos professores da rede pública de ensino de Macaé apontou escores críticos nas dimensões de Desgaste psíquico e Culpa. Os resultados indicaram que os 
professores estão intensamente submetidos a estresse, especialmente as mulheres, os mais jovens e os que trabalhavam há menos de 15 anos no magistério. Cabe destacar que, caso haja persistência dos estressores, a SB pode surgir associada a uma piora da qualidade de vida dos professores e do trabalho que desempenham.

O estresse laboral dificilmente será eliminado completamente. Contudo, esse pode ser amenizado a níveis controláveis para que os professores possam concomitantemente desempenhar suas atividades laborais e preservar sua saúde. É de suma importância que os professores tenham conhecimento acerca das temáticas relacionadas ao equilíbrio entre as demandas do trabalho e da vida social. Também é essencial pensar em estabelecer metas realistas e desenvolver estratégias para o enfrentamento e prevenção do estresse ocupacional.

O esgotamento dos professores é um tópico-chave, e os achados do presente estudo podem contribuir para o direcionamento de pesquisas futuras, bem como para a reflexão dos diferentes atores sobre a problemática da saúde de professores.

\section{REFERÊNCIAS}

ARAÚJO, T.M.; PINHO, S. P.; MASSON, M. L. Trabalho e saúde de professoras e professores no Brasil: reflexões sobre trajetórias das investigações, avanços e desafios. Cadernos de Saúde Pública; 35 Sup 1: e00087318, 2019.

ARVIDSSON, I; HÅKANSSON, C.; KARLSON, B; BJÖRK, J; PERSSON, R. Burnout among Swedish school teachers - a cross-sectional analysis. BioMed Central Public Health, v. 16, n. 823, p. 1-11, 2016.

ASSUNÇÃO, A. A.; ABREU, M. N. S. Pressão laboral, saúde e condições de trabalho dos professores da Educação Básica no Brasil. Cadernos de Saúde Pública, v. 35, n. suppl 1, 2019.

BATISTA, J. B. V.; CARLOTTO, M. S.; COUTINHO, A. S.; AUGUSTO, L. G. S.

Prevalência da Síndrome de Burnout e fatores sociodemográficos e laborais em professores de escolas municipais da cidade de João Pessoa, PB. Revista Brasileira de Epidemiologia, v. 13, n. 6, p: 502-512, 2010.

BAtista, J. B. V; CARlOTtO, M. S.; COUTINHO, A. S.; AUGUSTO, L.G. S. Síndrome de Burnout: confronto entre o conhecimento médico e a realidade das fichas médicas. Psicologia em Estudo, Maringá, v. 16, n. 3, p. 429-435, 2011.

CARLOTTO, S. M.; LIBRELOTTO, R.; PIZZINATO, A.; BARCINSKI, M. Prevalência e factores associados à Síndrome de Burnout nos professores de ensino especial. Análise psicológica, v. 30, n. 3, p. 315-327, 2012. 
CARLOTTO, S. M.; PALAZZO, L. S. Síndrome de Burnout e fatores associados: um estudo epidemiológico com professores. Cadernos de Saúde Pública, Rio de Janeiro, v. 22, p. 1017 1026, 2006.

CARLOTTO, S. M. Prevenção da Síndrome de Burnout em professores: um relato de experiência. Psicologia da Saúde, Rio Grande do Sul, v. 22, n. 1, p. 31-39, 2014.

CARLOTTO, S. M. Síndrome de Burnout em Professores: Prevalência e Fatores Associados. Psicologia: Teoria e Pesquisa, v. 27 n. 4, p. 403-410, 2011.

CARVALHO, F. A. A exaustão docente: subsídios para novas pesquisas sobre a Síndrome de Burnout em professores. Estudos e Pesquisa em Psicologia, Rio de Janeiro, v. 2, n. 2, p. 1-14, 2002.

CONNELL, R. Masculinidade corporativa e o contexto global: um estudo de caso de dinâmica conservadora de gênero. Cadernos Pagu, n. 40, p. 322-344, 2013.

CUNHA, K. W. V. A produção científica no Brasil nos anos de 2003 a 2008 sobre Síndrome de Burnout e docência. 57 f. Dissertação (Mestrado em Saúde Pública) - Escola Nacional de Saúde Pública Sérgio Arouca, Rio de Janeiro, 2009.

FRANÇA, T. L. B.; OLIVEIRA, A. C. B. L.; LIMA, L. F.; MELO, J. K. F.; SILVA, R. A. R. Síndrome de Burnout: Características, diagnóstico, fatores de risco e prevenção. Revista de Enfermagem UFPE OnLine, Recife, v. 8, n. 10, p. 3539-3546, 2014.

FRANCO, C. L.; MONTEIRO, S. P. Padrão de consumo de álcool e tabaco entre professores universitários. Revista Baiana de Enfermagem, Salvador, v. 30, n. 2, p. 1-11, 2016.

FREUDENBERGER, H. J. „Staff burn-out“. Journal of Social Issues, Washington, v. 30, n. 1, p. 159-165, 1974.

FRIEDRICH, D. C. A.; MACEDO, F; REIS, H. A. Vulnerabilidade ao stress em adultos jovens. Revista Psicologia Organizações e Trabalho, v. 15, n. 1, p. 59-70, 2015.

GASPARINI, S. M.; BARRETO, S. M.; ASSUNÇÃO, A. A. O professor, as condições de trabalho e os efeitos sobre sua saúde. Educação e Pesquisa, v. 31, n. 2, p. 189-199, 2005.

GAVISH, B.; FRIEDMAN, I. A. Novice teachers' experience of teaching: a dynamic aspect of burnout. Social Psychology of Education, V. 13, N. 2,P.141-167, 2010.

GIL-MONTE, R. P.; CARLOTTO, S. M.; CÂMARA, G. S. Validação da versão brasileira do "Cuestionario para la Evaluación del Síndrome de Quemarse por el Trabajo"em professores. Revista de Saúde Pública, v. 44, n. 1, p. 140-147, 2010.

GOULART JUNIOR, E; LIPP, N. E. M. Estresse entre professoras do ensino fundamental de escolas públicas estaduais. Psicologia em Estudo, v. 13, n. 4, p. 847-857, 2008.

GUIRALDELLI, R. Adeus à divisão sexual do trabalho?: desigualdade de gênero na cadeia produtiva da confecção. Sociedade e Estado, v. 27, n. 3, p. 709-732, 2012. 
KAN, Y. M; SUlLIVAN, O; GERSHUNY, J. Gender Convergence in Domestic Work: Discerning the Effects of Interactional and Institutional Barriers from Large-scale Data. Sociology, v. 45, n. 2, p. 234-251, 2011.

KOETZ, L.; REMPEL, C.; PÉRICO, E. Qualidade de vida de professores de Instituições de Ensino Superior Comunitárias do Rio Grande do Sul. Ciência \& Saúde Coletiva, v. 18, p. 1019-1028, 2013.

MATTOS, R. D. S.; LUZ, M. T. Sobrevivendo ao estigma da gordura: um estudo socioantropológico sobre obesidade. Physis Revista de Saúde Coletiva, v. 19, p. 489-507, 2009.

NOGUEIRA, R. P. Higiomania: a obsessão com a saúde na sociedade contemporânea. In: VASCONCELOS, Eymard Mourão. A saúde nas palavras e nos gestos: reflexões da rede de educação popular e saúde. São Paulo: Hucitec, p. 63-72, 2001.

OLIVEIRA FILHO, A; NETTO-OLIVEIRA, E. R.; OLIVEIRA, A. A. B. Qualidade de vida e fatores de risco de professores universitários. Revista de Educação Física, v. 23, n. 1, p. 57 67, 2012.

OLIVEIRA, P. A; ASSIS, M.; VILAÇA, M.; ALMEIDA, M. N. Os "pesos" de ser obeso: traços fascistas no ideário de saúde contemporâneo. Movimento, Porto Alegre, v. 18, n. 04, p. 99-119, 2012.

PADILLA, M. A; THOMPSON, J. N. Burning Out Faculty at Doctoral Research Universities. Stress Health, v. 32, n. 5, p. 551- 558, 2016.

PEREIRA, E. F; TEIXEIRA, C. S; ANDRADE, R. D.; SILVA-LOPES, A. O trabalho docente e a qualidade de vida dos professores na educação básica. Revista de Salud Pública, v.16, n.2, p. 221-231, 2014.

RAUSH, R. B.; DUBIELLA, E. Fatores que promoveram mal ou bem-estar ao longo da profissão docente na opinião de professores em fase final de carreira. Revista Diálogo Educacional, v. 13, n. 40, p.1041-1061, 2013.

SIMÕES, C. E. Investigação de esgotamento físico e emocional (burnout) entre professores usuários de um hospital público do município de São Paulo. 107 f. Dissertação (Mestrado em Ciências) - Faculdade de Saúde Pública, Universidade de São Paulo, São Paulo 2014.

SISTO, F. F.; BAPTISTA, M. N.; SANTOS, A. A. A.; NORONHA, A. P. P. Análise Fatorial da Escala de Vulnerabilidade ao Estresse no Trabalho (EVENT). Psicologia para América Latina. n.15, 2008.

WILHELM, F. A. X; ZANELLI, C. J. Características das estratégias de enfrentamento em gestores universitários no contexto de trabalho. Psicologia Argumento, Curitiba, v. 32, n. 79, p. 39-48. 2014.

ZIBETTI, M. L. T.; PEREIRA, S. R. Mulheres e professoras: repercussões da dupla jornada nas condições de vida e no trabalho docente. Educar em Revista, Curitiba, n. especial 2, p. 259-276, 2010. 